\title{
Aberration-Corrected Scanning Transmission Electron Microscopy of Single Crystals and Chemically-Gradient NMC Cathodes
}

\author{
Soroosh Sharifi-Asl ${ }^{1}$, Guoying Chen ${ }^{2}$, Jason Croy ${ }^{3}$, Mahalingam Balasubramanian $^{4}$, Reza Shahbazian- \\ Yassar $^{1}$ \\ 1. Mechanical and Industrial Engineering Department, University of Illinois at Chicago, Chicago, IL, \\ U.S. \\ 2. Energy Storage \& Distributed Resources Division, Lawrence Berkeley National Laboratory, Berkeley, \\ CA, U.S. \\ 3. Chemical Sciences and Engineering Division, Argonne National Laboratory, Lemont, IL, U.S. \\ 4. X-ray Science Division, Argonne National Laboratory, Lemont, IL, U.S.
}

Layered oxide cathode materials, with $\mathrm{R} 3 \mathrm{~m}$ hexagonal structure are known as the most important cathodes used in various scales of Li-ion batteries. Recently, $\mathrm{Li}\left(\mathrm{Ni}_{\mathrm{x}} \mathrm{Mn}_{\mathrm{y}} \mathrm{Co}_{\mathrm{z}}\right) \mathrm{O}_{2}$ cathodes known as NMC, with high theoretical specific capacity $\left(\sim 270 \mathrm{mAhg}^{-1}\right)$ and cost effectiveness have gained tremendous attention specially for large scale applications such as electric vehicles (EVs). However, rapid capacity fading, resulting from parasitic surface reactions such as transition metal dissolution, structural rearrangement and oxygen dissociation at high cut-off voltages limits the practical capacity of these cathodes to below $170 \mathrm{mAhg}^{-1}$ [1]. Therefore, many research efforts have been focused to understand the degradation mechanism of NMC cathodes. For instance, by electrochemical characterizations, X-ray spectroscopy/imaging and transmission electron microscopy [2,3], it was understood that, excessive Li-removal at high cut-off voltages results in formation of spinel and rocksalt phases, with low electrochemical activity and ionic conductivity, which leads to poor capacity retention. To resolve this issue, various surface modification approaches, such as coating with chemically robust metal oxides (such as $\mathrm{Al}_{2} \mathrm{O}_{3}$ and $\mathrm{TiO}_{2}$ ), or surface doping with stabilizing elements (such as $\mathrm{Al}$ and $\mathrm{Zr}$ ) has been proposed .However, the results obtained from cathodes with surface protection coating are sometimes controversial, in addition sample uniformity and reproducibility of the results are often challenging. Also, many coating techniques are expensive and time-consuming so cannot be scaled up to meet industrial requirements. Therefore, the urge for a cost-effective and viable solution to these challenges has driven the researchers towards synthesis of single-crystalline and chemically-gradient NMC cathodes.

Single-crystal NMC cathodes, composed of micron-size single-crystals have many benefits compared to poly crystalline NMC cathodes. Unlike conventional poly-crystalline NMC cathodes that are composed of agglomerates of many nano-meter size grains of primary particles, single crystal NMCs have much less exposed surface area, which alleviates the parasitic surface reactions [4]. In addition, absence of structural defects such as grain boundaries allows for smooth Li intercalation. Another attractive design to control the surface reactions, is synthesis of chemically-gradient cathode particles with higher extent of stable elements at the surface and higher extent of electrochemical active elements at the core. This method allows for tuning the capacity and surface stability to obtain long lasting, high capacity cathode materials [5].

In this work, we utilized aberration-corrected scanning transmission electron microscopy to study the effect of composition on the structural stability of single-crystal NMC cathode. In this study, high angle annular dark field (HAADF) imaging together with electron energy loss spectroscopy was utilized to 
study the effect of increased Ni content on the defect formation, transition metal displacement, surface reconstruction and degradation of single crystalline and chemically-gradient NMC cathodes. Figure 1A, demonstrates an HAADF image from the surface of pristine single crystalline NMC particle with the low magnification image and the corresponding FFTs inset. From faceted surfaces and uniform contrast observed in the the low magnification image, the successful synthesis of single crystal particles can be realized. By performing the same experiments, together with EELS analysis, on the single crystal NMC cathodes with various $\mathrm{Ni}$ concentrations, we studied the effect of $\mathrm{Ni}$ content on the extent of surface reconstruction layer and transition metal migration in the structure of particles.

Similarly, we are utilizing aberration corrected transmission electron microscopy to characterize the composition and structure of chemically-gradient NMC cathodes. Chemical gradient of the cathode particles has been identified by EELS map analysis and the information has been input to the sample synthesis route to optimize the composition. An example of a set of EELS map results, taken from cathode particles with high $\mathrm{Ni}$ concentration at the surface has been shown in Figure 1. Figure 1B demonstrates the EELS map results from the sample treated at $900{ }^{\circ} \mathrm{C}$. It can be observed that $\mathrm{Ni}$ has a very week signal, which completely fades by moving $\sim 100 \mathrm{~nm}$ away from the surface of the sample. The Ni signal can be better visualized in the sum spectrum shown on top as a black spectrum. The EDS results, detects $1.5 \%$ of $\mathrm{Ni}$ in this particle which has penetrated gradually in the top $200 \mathrm{~nm}$ shell of the particle. By performing the same set of experiments on cathodes synthesized with lower annealing temperature, it can be concluded that, by decreasing the annealing temperature, the diffusion extent of $\mathrm{Ni}$ into the outer shell of cathode particle has decreased, and the density of the Ni has increased in the shell. Effect of $\mathrm{Ni}$ concentration on the atomic structure and surface reconstruction has also been investigated with atomic resolution imaging and EELS analysis.

[1] Jung, S.-K.; Gwon, H.; Hong, J., Adv. Energy Mater. 4 (2014), p. 1300787.

[2] Abraham, D. P.; Twesten, R. D.; Balasubramanian, M., Electrochem. Comm. 4 (2002), p. 620.

[3] Lin, F.; Markus, I. M.; Nordlund, D., Nat. Commun. 5 (2014), p. 3529.

[4] Li, J.; Cameron, A. R.; Li, H., J. Electrochem. Soc. 164 (2017), p. 1534.

[5] Koenig, G. M.; Belharouak, I.; Deng, H., Chem. Mater. 23 (2011), p. 1954.
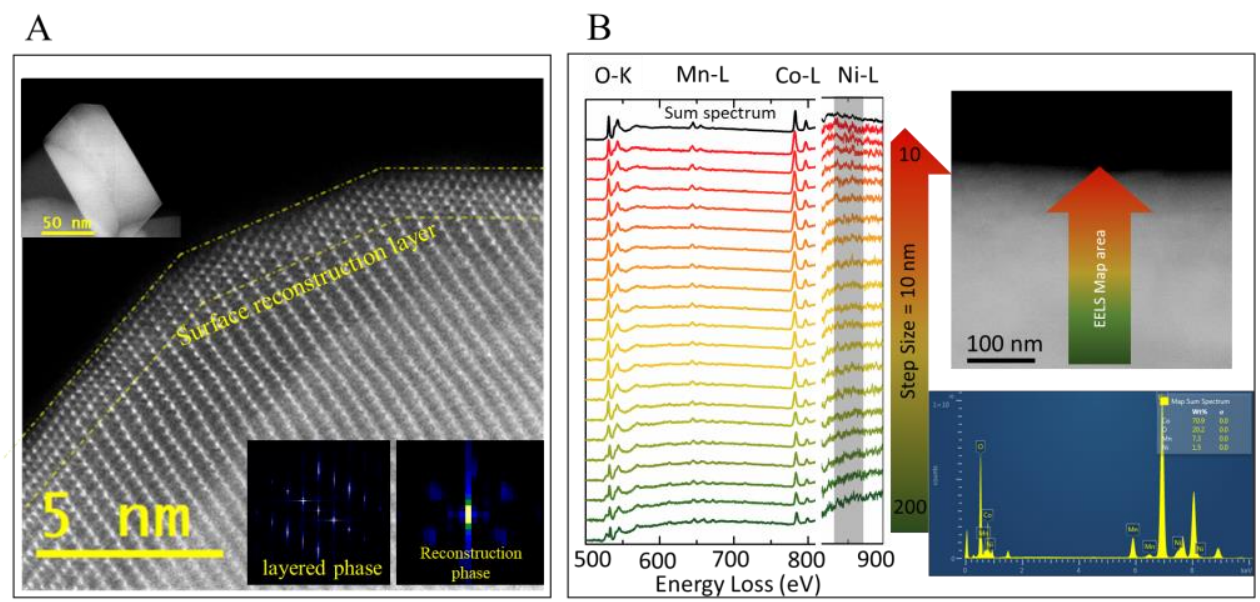

Figure 1. (A) Atomic resolution HAADF image from a single crystal NMC particle, showing the surface reconstruction layer, together with low magnification and FFT images inset. (B) EELS map results plotted as a function of distance from surface, obtained from a chemically-gradient cathode particle. 